Relations industrielles

Industrial Relations

\title{
L'accès à la syndicalisation
}

\section{Kenneth George}

Volume 41, numéro 3, 1986

URI : https://id.erudit.org/iderudit/050233ar

DOI : https://doi.org/10.7202/050233ar

Aller au sommaire du numéro

Éditeur(s)

Département des relations industrielles de l'Université Laval

ISSN

0034-379X (imprimé)

1703-8138 (numérique)

Découvrir la revue

Citer cet article

George, K. (1986). L'accès à la syndicalisation. Relations industrielles / Industrial

Relations, 41(3), 583-595. https://doi.org/10.7202/050233ar
Résumé de l'article

L'accès à la syndicalisation
Tous droits réservés (c) Département des relations industrielles de l'Université Laval, 1986
Ce document est protégé par la loi sur le droit d'auteur. L’utilisation des services d'Érudit (y compris la reproduction) est assujettie à sa politique d'utilisation que vous pouvez consulter en ligne.

https://apropos.erudit.org/fr/usagers/politique-dutilisation/ 


\section{L'accès à la syndicalisation}

\section{Kenneth George}

Au cours des 20 dernières années, les chefs syndicaux, les dirigeants d'associations patronales, les leaders politiques ont eu à intervenir de façon répétée - et quelquefois agressive - sur le problème de l'accès à la syndicalisation. C'est l'acuité du problème et, partant, la peur des leaders politiques de prendre position sur la question qui, pour plusieurs, explique la création de la Commission consultative sur le Travail (Commission Beaudry), commission qui a déposé son rapport à l'automne 1985; de fait, l'enjeu le plus important, aux yeux de tous les groupes qui ont présenté des mémoires devant la Commission Beaudry, c'était le problème de l'accès à la syndicalisation ${ }^{1}$.

Les universitaires québécois n'ont pas été indifférents à ce problème. Ils ont tenté de le préciser et de l'expliquer en fonction, non pas de normes démocratiques ou d'actes de foi, mais en référence à des phénomènes politiques, légaux, économiques et sociaux. L'objectif de cet article est de présenter une revue de la littérature scientifique québécoise sur le problème de l'accès à la syndicalisation.

Il importe de préciser que, dans cette littérature, le phénomène à expliquer, la variable dépendante, n'est pas toujours exprimé par le concept «accès à la syndicalisation» ou l'expression "difficulté d'accès à la syndicalisation». Certains auteurs traiteront de la saturation du taux de syndicalisation, d'autres de la propension des travailleurs à se syndiquer ou de l'opportunité de se syndiquer. Il ne faut certes pas minimiser l'importance du choix des termes, choix qui reflète des visions différentes de la réalité; on aura néanmoins saisi qu'on fait référence ainsi à autant de facettes de la réalité complexe que traduit le concept d'accès à la syndicalisation.

Les universitaires - surtout des professeurs de relations industrielles - ont attiré l'attention sur quatre types de variable qui pourraient avoir un impact sur l'accès à la syndicalisation: 1) la législation et les mécanismes institutionnels, 2) les caractéristiques et les attitudes des travailleurs, 3) le contexte économique et 4) les caractéristiques de l'entreprise.

* GEORGE, Kenneth, professeur, Université du Québec à Hull.

** Je veux remercier le professeur Gilles Laflamme (Université Laval) pour ses commentaires sur une première version de ce ce texte.

1 Une synthèse des positions présentées par les groupes devant la Commission Beaudry est disponible auprès de l'auteur; des extraits de cette synthèse sont parus dans Le Devoir, 9 janvier 1986, p. 7.

Relat. Ind., vol. 41, no 3, 1986 () PUL ISSN 0034-379 X 


\section{LA LÉGISLATION ET LES MÉCANISMES INSTITUTIONNELS}

Nombre d'analystes se sont arrêtés à la question de l'impact de la législation et des mécanismes institutionnels sur l'accès à la syndicalisation. Deux types distincts de législation retiennent l'attention: la législation sur les relations du travail proprement dites et la législation normative.

\section{La législation sur les relations du travail}

Généralement, les auteurs proposent que cette législation peut avoir un effet sur l'expansion du syndicalisme mais que cette variable ne peut être isolée du contexte politique dont elle est l'expression.

À partir de l'expérience québécoise, Rondeau et Bélanger affirment que l'accès à la syndicalisation est étroitement associé au cadre législatif. Les auteurs suggèrent que ce facteur a surtout un effet sur l'opportunité de se syndiquer, comme le démontreraient les «faits historiques»:

Ce n'est pas un accident historique que la syndicalisation s'est accrue avec la libéralisation des lois relatives au droit d'association et aux relations du travail ${ }^{2}$.

Les auteurs ne disent mot cependant sur les contextes politiques ou sociaux qui amènent les diverses législations, ni sur les variations de cet impact dans le temps.

Pour Gilles Laflamme, la thèse de Rondeau et Bélanger et de tous ceux qui suggèrent une relation entre la législation et le taux de syndicalisation résiste peu à l'analyse des données empiriques et repose sur des fondements théoriques problématiques.

Pour Laflamme, poser la question de l'impact du régime des relations du travail sur l'accès à la syndicalisation, c'est-à-dire «croire à une relation causale implicite» entre ces deux facteurs, c'est quelque peu simplifier les choses. En effet,

on ne peut répondre convenablement à cette question sans se situer dans une perspective historique, privilégiant alors une démarche qui veut que tout régime de relations du travail se construit historiquement... (et) que les traits essentiels de notre régime sont le résultat de rapports sociaux et aussi le reflet de valeurs véhiculées dans la société3.

Laflamme s'en prend aussi à l'argumentation empirique souvent utilisée par ceux qui veulent démontrer une relation entre la législation et le taux de syndicalisation. Pour démontrer que le régime des relations du travail est déterminant sur le taux de syndicalisation, on compare souvent le faible taux de syndicalisation au Québec au fort taux (70\%) qu'on retrouve

2 Claude RONDEAU et Jacques BELANGER, «Le syndicalisme dans l'entreprise: tendances récentes et analyse», J. Bélanger, J. Boivin, C. Rondeau, J. Sexton, éditeurs, La syndicalisation dans le secteur privé au Québec, Québec, PUL, 1983, p. 29.

3 Gilles LAFLAMME, «L'impact du régime de relations du travail sur la syndicalisation», J. Bélanger et ali, op. cit., p. 79. 
en Suède, en Autriche ou en Belgique. Compte tenu du caractère sectoriel, régional ou national des négociations dans ces pays européens, caractéristiques différentes du régime québécois, on conclut à une relation de cause à effet entre le régime de relations du travail et l'accès à la syndicalisation.

Cet argument est problématique selon Laflamme parce qu'il ne tient pas compte du fait que, dans d'autres pays européens où la négociation est aussi régionale, sectorielle ou nationale, (des pays comme la France et l'Allemagne), les taux de syndicalisation sont faibles: $25 \%$ pour la France et $40 \%$ pour l'Allemagne. A elles seules, ces données permettent à tout le moins de nuancer l'argument habituel.

C'est donc ailleurs que dans le régime de relations du travail qu'il faut chercher l'explication, selon Laflamme, de la différence entre les cas de hauts taux de syndicalisation et le cas québécois. Pour l'auteur, c'est le type de syndicalisme pratiqué qui est la variable explicative déterminante, en particulier les caractéristiques suivantes du syndicalisme européen: son accent sur les luttes politiques, en plus des luttes économiques, et son insistance sur une formulation idéologique précise de ses orientations. Ces caractéristiques différencient le syndicalisme européen de notre expérience syndicale et, mieux que le régime de relations du travail, ces caractéristiques, combinées à d'autres, expliqueraient l'écart entre les taux de syndicalisation sur les deux continents.

Contrairement à Rondeau et Bélanger - et bien d'autres - Laflamme propose qu'il n'y a pas de justification théorique ou de preuve empirique permettant d'établir une relation entre la législation et le taux de syndicalisation. Selon l'analyste, d'autres sortes de variables seraient plus pertinentes pour expliquer les différentes entre les taux de syndicalisation qu'on retrouve dans quelques pays européens et le taux québécois de syndicalisation.

Par rapport aux thèses de ces auteurs, Hélène LeBel prend une position nuancée. Elle reconnaît d'abord le bien-fondé de l'argument de Laflamme à l'effet que les législations sur les relations du travail ne font que consacrer des pratiques existantes, des forces sociales particulières: une loi n'est pas faite dans un vacuum politique. Elle précise par ailleurs que cela ne doit pas empêcher de reconnaître que ces législations ont eu, dans l'immédiat, un impact positif réel et important sur la syndicalisation, en légitimant et légalisant les pratiques syndicales et en enlevant quelques obstacles à la syndicalisation. La thèse de Rondeau et Bélanger serait donc juste pour la période qui suit immédiatement la législation:

(il) serait absurde de prétendre que le Wagner Act américain ou notre Loi des relations ouvrières ou le Code du travail n'ont pas servi les syndicats ou favorisé leur expansion ${ }^{4}$.

Cela dit, LeBel suggère aussi que ces bénéfices immédiats n'allaient pas sans un certain prix. C'est qu'en légalisant une pratique, la loi allait limiter l'expansion du syndicalisme aux lieux où cette pratique aurait un sens:

4 Hélène LEBEL, "Commentaires», dans J. Bélanger et al., op. cit., p. 91. 
Un syndicalisme axé principalement sur la revendication économique au sein d'une entreprise ou d'un établissement n'est-il pas presque inévitablement restreint à ces secteurs d'activités où la revendication économique est possible eu égard aux circonstances de l'entreprise et susceptible d'être efficace à cause de l'existence d'un rapport de forces au moins raisonnablement équilibré? ${ }^{5}$.

En d'autres mots, LeBel suggère que l'impact permanent de la législation est de limiter l'univers des entreprises où la syndicalisation est possible. Là où la pratique syndicale qui a justifié la législation est inappropriée, l'accès à la syndicalisation serait rendu plus difficile.

C'est un thème semblable que développe Gérard Dion quand il suggère qu'on a fait erreur, dans notre système juridique, de ne pas reconnaître les différences essentielles entre la grande entreprise industrielle et les autres types d'entreprise:

Dans la petite entreprise où tout le monde se connaît, où des liens personnels existent tant chez les travailleurs entre eux qu'entre les membres de la direction et les travailleurs eux-mêmes et enfin où la conscience de la viabilité de l'entreprise est communément ressentie d'une façon quotidienne, on n'a pas à être surpris que les appels à la grande solidarité avec tous les travailleurs provenant des centrales syndicales restent souvent sans échos ${ }^{6}$.

Compte tenu du caractère essentiellement différent des relations du travail dans la grande entreprise et la PME, ce n'est pas favoriser la syndicalisation des travailleurs que d'imposer à la $\mathrm{PME}$ un régime juridique propre à la grande entreprise.

La conclusion de LeBel et Dion quant à l'effet de la législation sur le type de lieux de travail où la syndicalisation peut se faire a déjà été présentée par Léo Roback et Jean Bernier qui, tous deux, démontrent, à partir d'unités d'analyses différentes, que la législation québécoise rend difficile l'accès à la syndicalisation dans les PME.

L'unité d'analyse de Roback, ce sont les accréditations accordées. En 1971, 1401 accréditations ont été accordées, suivant les critères de l'époque (50\% de salariés ayant signé une carte). En 1972, c'est 1297 accréditations qui ont été accordées et, dans les 12 mois entre avril 1974 et mars 1975 , 1223 . La taille moyenne des unités pour chacune de ces trois périodes était 23, 30 et 34 travailleurs. De ces données, Roback conclut:

Il est clair que le régime de l'accréditation par établissement ou par «employeur» permet à quelques dizaines de milliers de travailleurs de se syndiquer. Il demeure, toutefois, que ce processus est lent, et que l'accroissement du nombre des syndiqués au rythme de 30 ou 40 mille nouveaux syndiqués par an, ne fait que correspondre à l'accroissement de la main-d'oeuvre salariée. Le degré de pénétration syndicale, à moyen terme, reste donc inchangé $e^{7}$.

5 Ibid., son souligné.

6 Gérard DION, «Pourquoi et comment accroître la syndicalisation dans le secteur privé?», J. Bélanger et alii, op. cit., p. 165.

7 Léo ROBACK, La syndicalisation sectorielle: pour une solution a l'organisation des non-syndiqués, Montréal, IRAT, bulletin No 10, 1977, p. 22. 
Cette question de la relation entre la pénétration syndicale et la taille des unités de négociation a aussi été étudiée par Bernier. Comme Roback, Bernier démontre que «la majorité des conventions sont conclues dans des unités de petite taille... ces dernières sont à ce point petites qu'elles ne regroupent qu'une faible proportion de salariés visé par des conventions» ${ }^{8}$.

Bernier arrive à cette conclusion à partir de l'étude de 1930 conventions collectives en vigueur à l'été de 1973 et couvrant 15 employés et plus, à l'exception des conventions d'Hydro-Québec et des secteurs public et parapublic. Ces conventions couvraient 271000 travailleurs.

Le tableau 1 le démontre, alors que $43,8 \%$ des conventions s'appliquent dans des entreprises de 15 à 49 salariés, ces conventions rejoignent moins de $10 \%$ des travailleurs couverts par les 1930 conventions. Il ne semble donc pas y avoir de commune mesure entre l'effort investi pour négocier une convention et le nombre total de travailleurs affectés, ce que démontre aussi le fait que seulement $16,5 \%$ des conventions sont signées dans des entreprises de plus de 200 salariés, conventions qui s'appliquent à plus de $63 \%$ des travailleurs visés.

\section{TABLEAU 1}

Distribution des conventions collectives et des travailleurs visés en fonction de la taille des unités de négociation

Taille de l'unité
Conventions collectives

Nombre

846

460

306

222

97

1931
$\%$

43,8

23,8

15,9

11,5

5,0

100,0
Travailleurs

Nombre

$\%$

8,6

11,7

15,2

23,9

39,6

107327

100,0

Source: J. BERNIER, «La convention collective: un indicateur de la réalité syndicale québécoise», Relations Industrielles, Québec, vol. 29, no 1, 1974, p. 176.

Comme Roback, Bernier explique ce «morcellement incroyable des unités» par la législation et, en particulier, deux facteurs qui "conduisent» à ce morcellement: le Code du travail qui limite l'unité de négociation à un employeur et la «pratique québécoise en matière d'accréditation syndicale... qui conduit souvent à l'accréditation sur la base d'un établissement ou d'une fraction d'établissement»?.

8 Jean BERNIER, «La convention collective: un indicateur de la réalité syndicale québécoise", Relations industrielles, Québec, vol. 29, no 1, 1974, p. 176.

9 Idem., p. 177. 
Comme on l'a dit plus haut, dans la mesure où l'on accepte que la législation mérite de retenir l'attention comme variable indépendante déterminant le taux de syndicalisation, on peut conclure de la littérature que cette variable provoque, dans l'immédiat, une augmentation du taux de syndicalisation mais que, dans le moyen terme, elle oblige à une pratique qui nuit à l'expansion syndicale à l'extérieur de l'univers où cette pratique a été initiée et où elle est appropriée. Une fois fait le "plein» des travailleurs syndicables dans les grandes entreprises, il devient très difficile pour les organisations syndicales d'assurer leur expansion.

\section{La législation normative}

L'action législative de l'État qui peut avoir un impact sur la syndicalisation des travailleurs ne se limite pas à la définition des règles qui constituent le Code du travail. L'action normative de l'État a aussi un impact. Généralement, les analystes suggèrent que l'action normative nuit à l'expansion syndicale; par rapport à ce consensus général chez les scientifiques, il y a un analyste qui soutient la thèse contraire.

Faisant référence à la Loi sur la santé et la sécurité du travail, la Loi sur les normes $d u$ travail, etc... Laflamme, ${ }^{10}$ comme Dion, ${ }^{11}$ propose que la législation normative a un effet négatif sur le taux de syndicalisation en rendant moins nécessaire, aux yeux des travailleurs, la nécessité de se syndiquer:

L'État, qui devient l'État-Providence, par un ensemble de législations qui diminuaient les rapports de dépendance du salarié à l'égard de l'entreprise et du syndicat pour certaines conditions centrales de son existence et de celles de sa famille, a pu ainsi contribuer à rendre moins urgente la présence même du syndicat sur les lieux du travail ${ }^{12}$.

Comme le fait remarquer Laflamme, il ne s'agit là que d'une hypothèse. Les études empiriques n'ont pas encore été faites sur ce sujet.

Ce sont des hypothèses tout à fait contraires que propose André Côté. Côté ne croit pas que les interventions normatives de l'État nuisent «à moyen et à long terme» à la syndicalisation: «l'effet pourrait être tout à fait à l'opposé» ${ }^{13}$ et ceci pour trois raisons:

a) malgré l'action de l'État, il y a toujours une très grande marge entre les droits définis dans les lois normatives et ceux reconnus dans une convention collective, de sorte que les travailleurs auront toujours quelque chose à gagner de la syndicalisation;

10 Gilles LAFLAMME, op. cit.

11 Gérard DION, op. cit., pp. 154-171.

12 Gilles LAFLAMME, op. cit., p. 86.

13 André CÔTE, «Impacts des normes sur les rapports collectifs», Normes du travail: impacts sur la gestion des ressources humaines et sur les rapports collectifs du travail, Université de Montréal, XV Colloque des relations industrielles, 1984, p. 267. 
b) l'utilisation des recours définis dans les lois normatives nécessitera, pour être efficace, que les travailleurs puissent compter sur l'appui d'une certaine organisation;

c) la concertation des salariés et de l'employeur en entreprise, concertation à laquelle oblige souvent la loi pour l'élaboration et la mise en oeuvre des programmes, pourrait être le début d'un processus d'organisation des travailleurs. En effet, comme, via ces programmes, l'employeur et une instance administrative affecteront leurs conditions de travail, les salariés, "pris collectivement, (en viendront à avoir) un intérêt à se prévaloir des droits ou des possibilités d'intervention que la loi leur reconnaît»; $;^{14}$ or, pour ce faire, les travailleurs devront s'organiser et ils seront alors plus disposés à accueillir l'offre de services techniques (le know-how) des institutions syndicales.

En d'autres mots, non seulement l'action normative de l'État ne devrait pas nuire au développement du mouvement syndical, mais, en plus, elle devrait offrir des justifications nouvelles à la syndicalisation des travailleurs.

On est donc en présence de deux façons contraires de voir l'effet de la législation normative sur la propension des travailleurs à se syndiquer. Il importe de rappeler que toutes ces considérations, les auteurs le notent, sont quelque peu spéculatives; en effet, à ce jour, aucune de ces hypothèses n'a fait l'objet d'une recherche empirique.

\section{LES CARACTÉRISTIQUES ET ATTITUDES DES TRAVAILLEURS}

Un deuxième type de variable qui retient l'attention, c'est le travailleur lui-même. La littérature aborde la question de la relation entre les travailleurs et la syndicalisation de deux façons. Certains analystes comme Rondeau et Bélanger, Lorrain et Brunet proposent des relations de type universel entre des caractéristiques des travailleurs et leur propension a la syndicalisation. D'autres, comme Dion, tentent plutôt de caractériser les attitudes des travailleurs par rapport au mouvement syndical québécois.

Rondeau et Bélanger ${ }^{15}$ ne formulent pas d'hypothèse nouvelle sur l'effet des caractéristiques des travailleurs sur la propension à la syndicalisation. Plutôt, ils se contentent de résumer la littérature sur ce sujet en faisant part de leurs réserves:

a) la propension à la syndicalisation serait plus grande chez les jeunes; mais cette généralisation ne s'appliquerait pas aux travailleurs plus âgés parce que la syndicalisation permettrait une reconnaissance de leur ancienneté;

b) la propension à la syndicalisation serait moins grande chez les femmes que chez les hommes; mais cette généralisation - dont la logique n'est pas évidente - ne cacherait-elle pas plutôt le fait que les femmes, plus que les hommes, occupent des postes plus difficilement syndicables (emplois temporaires, à temps partiel, dans les services, etc...)?;

14 Idem., p. 268.

15 Claude RONDEAU et Jacques BELANGER, op. cit., pp. 28-29. 
c) enfin, la propension à se syndiquer augmente avec la hausse des salaires; mais ne peut-on pas plutôt penser que c'est la syndicalisation qui entraîne les hausses de salaires?

C'est autrement que Gérard Dion analyse les attitudes des travailleurs québécois par rapport à la syndicalisation. Les considérations de Dion s'inscrivent dans une tentative d'explication des difficultés qu'ont les centrales syndicales québécoises à recruter des membres.

Dion suggère d'abord qu'on ne saurait se limiter à des considérations sur les structures légales ou la conjoncture économique; il faut aussi tenir compte

d'un réel problème de désaffection des travailleurs syndiqués vis-à-vis du syndicalisme et même une véritable hémorragie causée par le nombre croissant de désaffiliation et de demande en révocation d'accréditation syndicale ${ }^{16}$.

Dans ce dernier cas, la situation devrait être d'autant plús préoccupante qu'elle met en cause des travailleurs qui devraient avoir expérimenté les bienfaits du syndicalisme.

Cette désaffection des travailleurs pour le syndicalisme serait liée, selon Dion, à trois facteurs:

a) l'incapacité des syndicats de faire leur auto-critique, de s'ajuster au nouveau contexte économique et même de voir qu'il y a un problème de désaffection - problème que les syndicats tiennent à expliquer «uniquement par des machinations externes, des collusions antisyndicales et l'inadéquation de la législation"; ${ }^{17}$

b) la législation normative qui rend moins nécessaire, aux yeux des travailleurs, la syndicalisation;

c) l'attitude des syndicats dans le secteur public, «particulièrement avec les grèves d'hôpitaux et du transport en commun" ${ }^{18}$.

Bref, pour Dion, les difficultés de syndicalisation des travailleurs ne sauraient s'expliquer sans référence à la faible volonté des travailleurs de se syndiquer et cette attitude, précise-t-il, n'est pas indépendante de la protection offerte par l'État et du type d'expérience syndicale vécue au Québec, plus précisément de l'image publique du syndicalisme.

Cette relation qu'établit Dion entre la syndicalisation des travailleurs et l'image publique que projetteraient les organisations syndicales ne correspond pas tout à fait aux données recueillies par Jean Lorrain ${ }^{19}$ sur le comportement des travailleurs lors des votes d'accréditation syndicale; les travailleurs votent en faveur de l'accréditation s'ils perçoivent que la syndicalisation leur offrira des avantages personnels. La propension des travailleurs à se syndiquer serait donc reliée, non pas à l'image publique du

16 Gérard DION, op. cit., p. 167.

17 Idem., p. 168.

18 Idem., p. 169.

19 Jean LORRAIN, "L'analyse causale d'un modèle de la syndicalisation», Actes $d u$ congrès de l'Association des sciences administratives du Canada, 1983. 
syndicalisme, mais plutôt à la perception que les travailleurs ont de l'utilité du syndicat comme moyen d'améliorer leurs conditions immédiates de travail. C'est la dimension concrète, «instrumentale» du syndicalisme qui importerait davantage que l'image publiquement projetée.

Par ailleurs, à partir d'une étude faite par Jean Lorrain et Luc Brunet sur les facteurs qui amènent des cadres d'entreprises à juger que la syndicalisation pourrait améliorer leur sort, on peut conclure que le «climat organisationnel» d'une entreprise joue un rôle important dans le développement des perceptions du syndicalisme. Ainsi, plus le climat de travail est «froid, ridige et bureaucratique», ${ }^{20}$ moins les gestionnaires manifesteront d'intérêt pour les opinions et idées des travailleurs, plus ceux-ci développeront une perception utilitaire ou «instrumentale» du syndicat.

Postulant implicitement une volonté universelle de la part des travailleurs de se syndiquer, Rondeau et Bélanger considèrent uniquement les caractéristiques qui entraînent une plus grande propension à se syndiquer. Dion s'interroge sur la prémisse des deux auteurs; cette volonté ne serait pas universelle et toute analyse des difficultés de syndicalisation doit tenir compte de l'intensité de la volonté des travailleurs de se syndiquer, intensité qui peut varier avec le sentiment que les travailleurs ont du besoin de se syndiquer et de l'image projetée par les syndicats. Lorrain et Brunet enfin, suggèrent que les travailleurs se syndiquent quand ils perçoivent la valeur instrumentale de la syndicalisation et que cette perception est déterminée principalement par le climat organisationnel de l'entreprise.

\section{LE CONTEXTE ÉCONOMIQUE}

On s'en doute bien, le contexte économique n'est pas sans impact sur l'accès à la syndicalisation. Rondeau et Bélanger suggèrent que l'inflation a un effet positif sur la syndicalisation alors qu'un taux élevé de chômage peut avoir un effet positif ou négatif. Les auteurs précisent ainsi la relation entre ces variables:

a) en situation d'inflation, la propension à se syndiquer et le taux de syndicalisation augmentent, le salarié cherchant à protéger son salaire réel et le patron ayant moins de raisons de s'opposer aux demandes des travailleurs, compte tenu des ventes et des profits supérieurs en situation d'inflation;

b) quand le niveau de chômage est élevé, le taux de syndicalisation n'augmente pas, la récession provoquant une résistance plus grande des employeurs à la syndicalisation et, partant, des risques plus grands pour les travailleurs qui chercheraient à se syndiquer;

c) par contre, «en période de chômage élevé, l'incitation à se syndiquer peut demeurer forte si le salarié y voit un moyen d'assurer sa sécurité d'emploi» ${ }^{21}$.

20 Jean LORRAIN et Luc BRUNET, «Climat organisationnel, satisfaction au travail et perception du syndicalisme", Relations industrielles, vol. 39, no 4, 1984, p. 675.

21 C. RONDEAU et J. BÉLANGER, op. cit., p. 27. 
Les auteurs notent que ces hypothèses sont problématiques du fait qu'elles reposent sur des études empiriques canadiennes peu concluantes. En plus, elles sont moins utiles dans la conjoncture où l'inflation accompagne un haut taux de chômage.

Peu d'autres analystes québécois se sont arrêtés à la question de la relation entre le contexte économique et le taux de syndicalisation. Cela peut s'expliquer, en partie, du fait qu'on sait, à partir des études faites au Canada anglais et aux USA, le peu de promesses qu'offre ce type d'analyse. De l'ensemble des études faites en effet, il se dégage que les auteurs arrivent difficilement à identifier les caractéristiques particulières du contexte économique qui ont un effet sur la syndicalisation ou encore l'importance relative de chacune des caractéristiques du contexte économique. En d'autres mots, le concept de contexte économique est si large qu'il est impossible de l'opérationnaliser de façon convenable pour en mesure l'impact.

\section{LES CARACTÉRISTIQUES DE L'ENTREPRISE}

Autant il a semblé difficile, dans l'étude de l'impact du contexte économique, de déterminer l'effet spécifique de chacune des caractéristiques de ce contexte, autant cet exercice semble difficile quand il s'agit de considérer l'effet des caractéristiques spécifiques de l'entreprise. La plupart du temps, les auteurs proposent des corrélations qui intègrent diverses variables, diverses caractéristiques de l'entreprise (taille, secteur industriel, statut de travail et compétitivité); ce n'est pas de façon isolée mais ensemble, de façon presque cumulative, que ces facteurs ont un effet sur l'accès à la syndicalisation.

Ainsi, dans son analyse des conventions collectives en vigueur à l'été 1973, Jean Bernier constate que c'est dans le secteur d'activité secondaire qu'il y a le plus de conventions, d'unités d'accréditation et de travailleurs syndiqués. Comme le démontre le tableau $2,58,3 \%$ des travailleurs syndiqués et $62,4 \%$ des conventions signées sont du secteur secondaire alors que seulement $31,7 \%$ des travailleurs syndiqués et $32,3 \%$ des conventions sont du secteur tertiaire, le reste étant du secteur primaire. Par ailleurs, alors que les travailleurs du secteur secondaire ne représentent que $30,6 \%$ de la maind'oeuvre totale, ils forment $58,0 \%$ des travailleurs syndiqués. À l'inverse, les travailleurs du secteur tertiaire qui constituent $62,2 \%$ de la main-d'oeuvre totale ne forment que $31,7 \%$ des travailleurs syndiqués.

Selon Bernier, deux facteurs expliquent cette situation à savoir la taille plus petite des entreprises du secteur tertiaire et le fait que le syndicalisme s'y soit implanté plus tard.

Bernier note aussi que l'accès plus difficile à la syndicalisation dans le secteur tertiaire où les entreprises sont de petite taille n'est pas indépendant d'une autre caractéristique de ces entreprises: ces entreprises emploient surtout des cols blancs alors que la pénétration syndicale est beaucoup plus grande chez les cols bleus. Cette corrélation était annoncée par les données du tableau 1; 68\% des travailleurs couverts par une convention sont des secteur primaire et secondaire où prédominent les cols bleus. 
TABLEAU 2

Distribution des conventions collectives et des travailleurs couverts en fonction du secteur d'activité

\begin{tabular}{|c|c|c|c|c|c|c|}
\hline \multirow[t]{2}{*}{$\begin{array}{l}\text { Secteur } \\
\text { d'activité }\end{array}$} & \multicolumn{2}{|c|}{$\begin{array}{c}\text { Conventions } \\
\text { collectives }\end{array}$} & \multicolumn{2}{|c|}{ Travailleurs } & \multicolumn{2}{|c|}{$\begin{array}{c}\text { Main-d'oeuvre } \\
\text { totale }^{a}\end{array}$} \\
\hline & Nombre & $\%$ & Nombre & $\%$ & Nombre & $\%$ \\
\hline Primaire & 102 & 5,3 & 20086 & 10,3 & 137000 & 7,2 \\
\hline condaire & 1204 & 62,4 & 156940 & 58,0 & $582000^{\mathrm{b}}$ & 30,6 \\
\hline ertiaire & 624 & 32,3 & 85990 & 31,7 & $1181000^{c}$ & 62,2 \\
\hline
\end{tabular}

Source: J. BERNIER, «La convention collective; un indicateur de la réalité syndicale québécoise», Relations Industrielles, Québec, vol. 29, no 1, 1974, p. 165.

a) données regroupées à partir de: Le marché du travail au Québec, 1972, B.S.Q., 1973 , p. 33.

b) excluant 117000 travailleurs de la construction.

c) excluant 210000 salariés des secteurs public et para-public.

Le caractère compétitif d'un secteur industriel (le nombre relativement élevé d'établissements), la petite taille des entreprises et l'exercice de fonctions de type "col blanc» seraient donc autant de facteurs qui rendent plus diffícile l'accès à la syndicalisation.

Ces conclusions de Bernier sont aussi celles de Léo Roback. À partir de l'indice qu'en 1972-1973, sur les 86055 employeurs du Québec, seulement 5386 avaient signé 4055 conventions collectives et après avoir ventilé ces données par secteur industriel, Roback suggère que dans un secteur industriel compétitif, l'accès à la syndicalisation est rendu difficile; dans «l'immense majorité» des établissements d'un tel secteur industriel, il n'y a pas de convention collective.

Il est intéressant de noter que cette corrélation entre la dispersion ou la compétitivité d'une industrie et l'accès à la syndicalisation existe aussi même dans un secteur industriel où plus de $50 \%$ des travailleurs sont régis par une convention collective:

Dans le secteur de la fabrication, ... environ $60 \%$ des travailleurs sont régis par une convention collective, mais il y a un syndicat dans seulement un établissement sur quatre $^{22}$.

De cette dernière corrélation, Roback dégage aussi une relation entre la taille d'une entreprise et la syndicalisation. Même si les données disponibles ne permettent pas de connaître la réalité des entreprises de moins de 20 travailleurs, ${ }^{23}$ les données présentées amènent Roback à conclure à tout le

22 Léo ROBACK, op. cit., p. 19; ses soulignés.

23 Dans les données du BSQ utilisées par Roback, les unités révisées sont celles qui ont au moins 20 employés. 
moins que «la taille moyenne des établissements syndiqués est plus grande que celles des établissements non syndiqués ${ }^{24}$. L'extrapolation d'une corrélation positive entre les difficultés plus grandes d'accès à la syndicalisation et la petite taille d'une entreprise devient alors légitime et logique pour l'auteur.

Par ailleurs, à partir des données tant sur le secteur manufacturier que sur le secteur tertiaire privé, (en particulier, le commerce, l'hôtellerie, la restauration et le secteur "finances, assurance et immeuble»), Roback suggère que certains types de travail ont pour effet d'accentuer la relation déjà établie entre la compétitivité d'une industrie (et partant le nombre et la taille des entreprises) et l'accès à la syndicalisation.

Ainsi, «dans tous les secteurs», la pénétration syndicale est énormément plus faible parmi les employés de bureau que chez les $\left\langle\right.$ (cols bleus» ${ }^{25}$. Pas surprenant alors que la pénétration syndicale soit plus difficile dans le tertiaire privé que dans le secteur de la transformation.

Eu égard au secteur tertiaire, Roback laisse entendre que la syndicalisation risque d'être encore plus problématique compte tenu de l'importance que prennent certains statuts de travail dans le domaine des services, en particulier, le temps partiel et le travail occasionnel (statuts surtout de femmes). «Il semble évident, note Roback, que les syndicats... devront ajuster leur stratégie... d'organisation en fonction de cette situation relativement nouvelle mais probablement permanente ${ }^{26}$.

Comme Bernier, Roback n'isole pas une caractéristique particulière de l'entreprise pour la mettre en relation avec les possibilités d'accès à la syndicalisation. La taille de l'entreprise n'est pas indépendante d'autres caractéristiques, comme la compétitivité du secteur industriel et les statuts de travail; les difficultés d'accès à la syndicalisation s'expliquent en tenant compte de l'effet combiné de ces caractéristiques.

C'est une même approche que proposent enfin Rondeau et Bélanger quand ils suggèrent que le degré de concurrence sur le marché du produit, l'importance relative des coûts de la main-d'oeuvre par rapport aux coûts totaux de production, la taille de l'entreprise, l'importance d'un groupe de travailleurs dans le processus de production, bref, ce qu'ils appellent la technologie, exercent un effet important sur l'opportunité de se syndiquer. Les auteurs ne précisent pas la relation entre les variables indépendantes et la variable dépendante, se contentant de préciser que les premières

déterminent l'opportunité de se syndiquer par leurs effets, soit sur la résistance plus ou moins grande de l'employeur à la syndicalisation, soit sur le cout plus ou moins élevé de l'organisation d'un syndicat ${ }^{27}$.

Tel que dit plus haut, les analystes semblent suggérer que l'accès à la syndicalisation n'est pas déterminé par une caractéristique particulière 
d'une entreprise. Serait davantage important l'effet combiné de caractéristiques qu'on retrouve souvent ensemble dans un lieu où la syndicalisation est difficile: secteur industriel compétitif, entreprise de petite taille où les travailleurs sont cols blancs avec des statuts non traditionnels de travail.

\section{L'ÉTAT DE LA LITTÉRATURE SCIENTIFIQUE}

La législation québécoise a probablement eu un impact immédiat positif sur l'accès à la syndicalisation mais elle constitue, à moyen terme, un frein à la syndicalisation; la législation normative et certaines attitudes syndicales peuvent faire paraître moins utile la syndicalisation aux yeux des travailleurs. Diverses caractéristiques des travailleurs peuvent favoriser la propension à la syndicalisation, d'autres peuvent avoir un effet contraire. Dans une situation de récession économique, la syndicalisation peut être plus difficile que dans un contexte où les profits sont plus assurés. Enfin, l'accès à la syndicalisation est problématique pour les travailleurs de PME opérant dans des secteurs économiques compétitifs et où, de surcroît, les travailleurs sont des cols blancs avec des statuts non traditionnels de travail. Ces termes généraux résument quelque peu la littérature scientifique sur le problème à l'étude.

Une lecture le moindrement attentive des discours patronaux et syndicaux sur le problème de l'accès à la syndicalisation permet de dégager une «vision», patronale ou syndicale, du phénomène: les acteurs font preuve de cohérence dans l'évaluation des facteurs qui ont un impact sur l'accès des travailleurs à la syndicalisation. On ne peut pas parler en ces termes de la littérature scientifique; la communauté n'offre pas encore de vision cohérente, de théorie, sur le problème, ce qui est l'objectif de toute recherche scientifique.

Plutôt, la littérature scientifique d'ici - comme celle d'ailleurs, on s'en doute - offre deux types de considérations. Certains, comme Rondeau et Bélanger, Dion ou Côté, formulent des hypothèses qui, si elles paraissent logiquement plausibles, doivent encore subir l'épreuve de la confrontation à la réalité. D'autres, comme Roback, Bernier, Laflamme, Lorrain et Brunet s'engagent certes dans des recherches empiriques, mais à partir souvent d'une ou deux hypothèses, ce qui est nettement insuffisant pour en arriver à une théorie.

Certes, des recherches nombreuses seront encore nécessaires avant que l'on ne puisse parler d'une vision, d'une théorie scientifique sur le problème de l'accès à la syndicalisation; les propos des scientifiques qu'on a résumés ici permettent cependant des espoirs dans la mesure où l'attention est portée sur des variables dont on a tout à fait raison de croire qu'elles ont une portée réelle sur l'accès des travailleurs à la syndicalisation. 